\title{
Congruência Modular aplicada à Criptografia
}

\author{
Djair P. dos Santos Lindinês C. da Silva \\ Fernando V. Costa Júnior Elthon A. da S. Oliveira \\ Universidade Federal de Alagoas, UFAL, 57309-005, Arapiraca, AL \\ E-mail: djairpsc@hotmail.com, lindinescoleta@hotmail.com, \\ fernando.math@hotmail.com, elthon@arapiraca.ufal.br.
}

\section{$\underline{\text { RESUMO }}$}

Nos meios eletrônicos e internet existem várias informações que devem ser mantidas em sigilo, as quais somente o emissor e o destinatário podem saber. Um meio de garantir o sigilo da informação é a criptografia, palavra que vem do grego kryptós = "secreto" e gráphein = "escrita", que é o estudo das técnicas de codificação/decodificação de informações. A criptografia busca a melhor forma de transformar dados em material dificilmente decifrável através de processos puramente matemáticos. Uma das ferramentas mais comuns é o uso da Matemática Discreta para, através da Congruência Modular, trabalhar sobre um código numérico onde apenas quem tem a chave de descriptografar consegue ler a mensagem (Cf. [1]). Este trabalho objetiva mostrar a importância da criptografia, e como a Criptografia RSA funciona a partir da aplicação da Congruência Modular, exibindo sua construção e funcionamento.

Definição 1: Definimos a Função de Euler como sendo a função $\varphi: \mathbb{N} \rightarrow \mathbb{N}$, onde $\varphi(n)=\sharp\{k \in$ $\left.\mathbb{N}^{*} \mid k \leq n \wedge m d c(k, n)=1\right\}$.

Definição 2: Sejam $a, b \in \mathbb{Z}$ e $n$ um inteiro positivo. Dizemos que a é congruente a b módulo $n$, e escrevemos $a \equiv b(\bmod n)$, se, e somente se, $a-b$ é múltiplo de $n$.

Estas definições nos serão úteis para a construção da Criptografia RSA. Esta envolve um par de chaves: uma pública (que pode ser conhecida por todos) e uma privada (que deve ser mantida em sigilo). Toda mensagem cifrada usando uma chave pública só pode ser decifrada usando a respectiva privada.

Escolhe-se dois números primos gigantescos, $p$ e $q$, preferencialmente com mais de cem dígitos, e define-se $N=p$.q. Cada pessoa que utiliza o sistema escolhe um $k \in \mathbb{N}$, tal que $m d c(k, \varphi(N))=1$, e então obtém-se a chave pública, que será $(N, k)$, usada da seguinte forma:

$$
(\text { texto codificado }) \equiv(\text { texto original })^{k}(\bmod N)
$$

Para obter a chave privada $(N, t)$, que decodificará a mensagem, é necessário resolver a congruência $k t \equiv 1(\bmod \varphi(N))$. Tal chave é usada da seguinte maneira:

$$
(\text { texto original }) \equiv(\text { texto codificado })^{t}(\bmod N)
$$

Como apenas o emissor e o destinatário da mensagem tem acesso aos números $p$ e $q$, fica praticamente impossível descobrir a chave de decodificação, e tomar números cada vez maiores pode aumentar a segurança. Contudo, o método RSA possui limitações matemáticas e técnicas com relação à quantidade de dados que se pode armazenar. Além de que existem ataques capazes de descriprotografar as informações, como: o método da força bruta, que tenta descobrir todas as chaves privadas possíveis; o timing attack, que determina uma chave privada baseando-se no tempo que um computador leva para decifrar uma mensagem; e técnicas matemáticas para fatoração de $N$, para determinação de $\varphi(N)$ sem ter $p$ e $q$, ou para determinar $t$ sem ter $\varphi(N)$ (Cf. [2]).

Exemplo: Usando a criptografia RSA, vamos codificar a palavra AMIGO, e decodificá-la em seguida. O primeiro passo consiste em construir uma tabela que fará corresponder as letras do alfabeto a números. Escolhendo de maneira aleatória, vamos fazer a tabela da seguinte forma: 


\begin{tabular}{|c|c|c|c|c|c|c|c|c|c|c|c|c|c|c|c|c|}
\hline A & B & C & D & E & F & G & H & I & J & K & L & M & N & O & P & Q \\
\hline 10 & 11 & 12 & 13 & 14 & 15 & 16 & 17 & 18 & 19 & 20 & 21 & 22 & 23 & 24 & 25 & 26 \\
\hline
\end{tabular}

\begin{tabular}{|c|c|c|c|c|c|c|c|c|c|c|c|c|c|c|c|}
\hline $\mathrm{R}$ & $\mathrm{S}$ & $\mathrm{T}$ & $\mathrm{U}$ & $\mathrm{V}$ & $\mathrm{W}$ & $\mathrm{X}$ & $\mathrm{Y}$ & $\mathrm{Z}$ & Á & É & Í & Ó & Ú & À & \\
\hline 27 & 28 & 29 & 30 & 31 & 32 & 33 & 34 & 35 & 36 & 37 & 38 & 39 & 40 & 41 & 42 \\
\hline
\end{tabular}

Obs.: Foi destinado um número para o espaço, e a numeração começou do 10 para evitar ambiguidades.

Devemos transformar a mensagem-texto em mensagem-numérica. Para isso, utilizaremos a tabela. Assim, a mensagem-texto 'AMIGO' corresponde à mensagem numérica '1022181624'. Para simplificar as contas, escolheremos números pequenos para $p$ e $q$. Vamos fazer $p=5$ e $q=13$. Assim, $N=p \cdot q=$ $=65$. Aplicando a função de Euler, temos que $\varphi(N)=\varphi(65)=48$.

O próximo passo é escolher um número $k$ para compor a chave de codificação. Devemos ter que $1<k<\varphi(N)$ e $m d c(k, \varphi(N))=1$. Tomaremos $k=11$. Assim, a chave pública (de codificação) é $(65,11)$, e será usada da seguinte forma:

$$
(\text { texto codificado }) \equiv(\text { texto original })^{11}(\bmod 65)
$$

Separaremos o texto-numérico em blocos de dois dígitos para codificar a mensagem. A maneira de separar a mensagem não é única, mas deve-se tomar cuidado, pois é necessário evitar que o bloco inicie com zero, e devemos ter cada número $a_{i}$ do bloco $a_{i}<N$. Então: $b_{i} \equiv a_{i}^{k}(\bmod N)$, onde $a_{i}$ é o texto original e $b_{i}$ o texto codificado, $\operatorname{com} i=1,2, \ldots, 5$. Daí, $b_{1} \equiv 10^{11}(\bmod 65) \Rightarrow b_{1} \equiv 30(\bmod 65)$. Logo, 30 é a codificação de 10. De forma análoga, temos:

$b_{2} \equiv 22^{11}(\bmod 65) \Rightarrow b_{2} \equiv 03(\bmod 65) ; b_{3} \equiv 18^{11}(\bmod 65) \Rightarrow b_{3} \equiv 47(\bmod 65)$;

$b_{4} \equiv 16^{11}(\bmod 65) \Rightarrow b_{4} \equiv 61(\bmod 65) ; b_{5} \equiv 24^{11}(\bmod 65) \Rightarrow b_{5} \equiv 24(\bmod 65)$.

Portanto, a mensagem codificada é: $\begin{array}{lllll}30 & 03 & 47 & 61 & 19 .\end{array}$

Para encontrar a chave de decodificação, basta resolver a congruência $11 t \equiv 1(\bmod 65)$, que fornece $t=35$. Logo, a chave privada (de codificação) é (65,35), e será usada da seguinte maneira:

$$
(\text { texto original }) \equiv(\text { texto codificado })^{35}(\bmod 65)
$$

Para verificar que o método RSA funcionou, vamos decodificar a mensagem:

$a_{1} \equiv 30^{35}(\bmod 65) \Rightarrow a_{1} \equiv 10(\bmod 65) ; a_{2} \equiv 03^{35}(\bmod 65) \Rightarrow a_{2} \equiv 22(\bmod 65)$;

$a_{3} \equiv 47^{35}(\bmod 65) \Rightarrow a_{3} \equiv 18(\bmod 65) ; a_{4} \equiv 61^{35}(\bmod 65) \Rightarrow a_{4} \equiv 16(\bmod 65)$.

$a_{5} \equiv 19^{35}(\bmod 65) \Rightarrow a_{5} \equiv 24(\bmod 65)$

Perceba que a mensagem-numérica que as contas retornaram (10 2218 16 24) coincidem, de acordo com a tabela, com a mensagem-texto 'AMIGO', como queríamos.

A criptografia RSA não é a única, porém é uma das mais eficientes. Destacamos a RSA, mas nosso trabalho se estende a outras criptografias baseadas na Congruência Modular. Algumas ainda mais simples do que a RSA.

Palavras-chave: Matemática Discreta, Congruência Modular, Criptografia, RSA

\section{Referências}

[1] FREIRE, Benedito T. V. Notas de Aula - Teoria dos Números. Disponível em: <http://www.olimpiada.ccet.ufrn.br/wp-content/uploads/2013/08/NOTAS-DE-AULA_09.pdf>

Acesso em: 06 dez. 2013.

[2] LÓPEZ, Javier G.; MEURER, Lúcio A. Estudos dos Ataques Matemáticos ao RSA e Hipótese de Modificação do Algoritmo. Disponível em: «http://sedici.unlp.edu.ar/bitstream/handle/10915/22344/Documento_ completo.pdf? sequence=1» Acesso em: 09 abr. 2014.

[3] OLIVEIRA, Maykon C. de. Aritmética: criptografia e outras aplicações de congruências. 2013. 63p. Monografia (Mestrado). Universidade Federal de Mato Grosso do Sul, Campo Grande, 2013. 